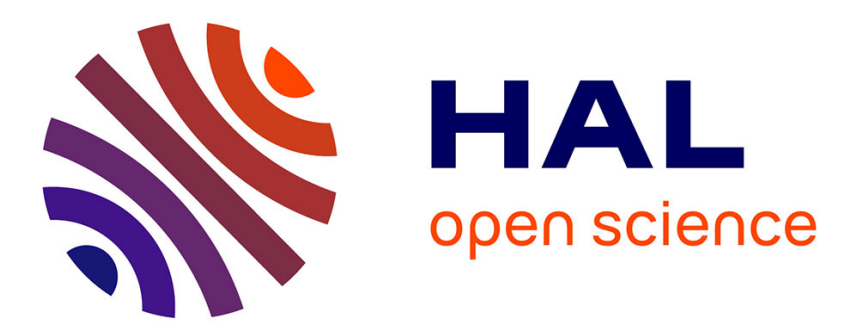

\title{
Pulsed jet phase-averaged flow field estimation based on neural network approach
}

Célestin Ott, Charles Pivot, Pierre Dubois, Quentin Gallas, Jérôme Delva, Marc Lippert, Laurent Keirsbulck

\section{- To cite this version:}

Célestin Ott, Charles Pivot, Pierre Dubois, Quentin Gallas, Jérôme Delva, et al.. Pulsed jet phaseaveraged flow field estimation based on neural network approach. Experiments in Fluids, 2021, 62 (4), pp.1-16. 10.1007/s00348-021-03180-0 . hal-03206636

\section{HAL Id: hal-03206636 https://hal.science/hal-03206636}

Submitted on 23 Apr 2021

HAL is a multi-disciplinary open access archive for the deposit and dissemination of scientific research documents, whether they are published or not. The documents may come from teaching and research institutions in France or abroad, or from public or private research centers.
L'archive ouverte pluridisciplinaire HAL, est destinée au dépôt et à la diffusion de documents scientifiques de niveau recherche, publiés ou non, émanant des établissements d'enseignement et de recherche français ou étrangers, des laboratoires publics ou privés. 


\title{
Pulsed jet phase-averaged flow field estimation based on neural network approach
}

\author{
Céletin Ott - Charles Pivot • Pierre Dubois - Quentin Gallas • Jérôme Delva • \\ Marc Lippert • Laurent Keirsbulck
}

Received: date / Accepted: date

\begin{abstract}
Single hot-wire velocity measurements have been conducted along a three-dimensional measurement grid to capture the flow-field induced by a $45^{\circ}$ inclined slotted pulsed jet. Based on the periodic behavior of the flow, two different estimation methods have been implemented. The first one, considered as the reference base-line, is the conditional approach which consists in the redistribution of the experimental data into spaceand time-resolved three-dimensional velocity fields. The second one uses a neural network to estimate $3 \mathrm{D}$ velocity fields given spatial coordinates and time. This paper compares the two methods for a complete flow-field estimation based on hot-wire measurements. Results suggest that the neural network is tailored to capture the phase-averaged dynamic response of the jet induced by the actuator, and identify the coherent structures in the flow field. Interesting performances are also observed when degrading the learning database, meaning that neural networks can be used to drastically improve the temporal or spatial resolution of a flow field estimation compared to the experimental data resolution.
\end{abstract}

Keywords Neural Network · Deep Learning · Actuators · Dynamic Characterization · Flow Control

C.Ott, C.Pivot, P.Dubois, Q.Gallas, J.Delva

Univ. Lille, CnRs, Onera, Arts et Métiers ParisTech, Centrale Lille, UMr CNRS 9014 - LMFL - Laboratoire de Mécanique des Fluides de Lille - Kampé de Fériet, F-5900 Lille, France

C.Ott, M.Lippert, L.Keirsbulck

LAmin, Umr CnRs 8201, Le Mont Houy, 59313 Valenciennes, France

E-mail: Laurent.Keirsbulck@uphf.fr

\section{Introduction}

Multidimensional flow field database development has become more and more important for a large number of applications (understanding of the physical phenomena involved Callaham et al. (ए2019), active flow control configuration optimisation Williams and MacMynowski (2012), parametric study for the prediction of specific phenomena occurrence Li et aL (2020)...) The main objective of flow field reconstruction consists in estimating the space- and time-resolved flow fields with the associated coherent structures. Experimentally, such a task is however challenging especially because of the technical complexity associated to the acquisition of experimental data directly resolved in space and time. To address these challenges, efforts can be made in the metrology process to capture the flow field and in the methodology to process experimental data. In the topic of flow field measurements, the stake is to capture the physics and the coherent structures in the flow field. Beyond the temporal resolution, the deployed metrology technique must also have a sufficient spatial resolution. Only a few metrology techniques allow to measure directly and vectorially a volumic velocity field, such as V3V or HPIV (space-time resolved particle tracking techniques on a thick laser sheet Soria and Atkinson ([2008)). These methods are particularly suitable for fluidic actuator's dynamic characterization as shown by Cambonie et al. (2013) and Cambonie and Aider (2014). Such approaches however require a complex experimental setup, which is not necessarily compatible with standard geometric configurations for various reasons (obstruction, intrusiveness, high-speed laser power, seeding issues, optical access, near-wall resolution...) Poelma et al. (201]), Haack et al. (2008). In this case, other 
methods with a trade-off on the nature of the recovered information can be implemented:

- local time-resolved measurements, such as hot-wire sensor (Fernandez et al. (2018)) or Laser Doppler Anemometry (Bisgaard (1983)) which allow to capture velocity signals at high frequency $(20 \mathrm{kHz})$

- space-resolved flow field, such as 2D PIV (or StereoPIV on a laser sheet Foucaut et al. (200) ,Béra et al. (200I)) which make possible to obtain velocity fields space-resolved but at a lower frequency (conventionally $2 \mathrm{kHz}$ for high-speed PIV) and only in a two dimensions plan, or refractive index gradient-based methods (shadowgraphy Emerick et al. (2012), Schlieren or holography Olchewsky et al. (2019)) which enable the capture of pressure fields (or pressure gradient fields) but not velocity fields.

Since the goal of the dynamic characterization process is to obtain a volumic space-time resolved velocity field, the experimental data have to be manipulated (when Holographic-PIV (3D space-time-resolved) can not be used), flow field can be estimated using reconstruction methods (based on incomplete experimental data, supplemented or not with stochastic estimations).

in particular, Kovasznay (14.949) has developed a method to extract a velocity field resolved in space and time. This method can be adapted to be based on a one-, twoor three-dimensional measurement grid on where local time-resolved signals are successively captured and synchronized using a noticeable trigger representative of the studied phenomenon period Ostermann et al. (2017). In the context of periodic fluidic actuators, this approach is particularly tailored and was adapted for various applications, mainly to convert local hot-wire measurements into:

o instantaneous velocity profiles Aeschlimann et al. (2013)

- 2D instantaneous velocity fields Kaman et al. ([T.9.9), Chovet et al. (2016), Ott et al. (2019a)

- 3D instantaneous velocity fields Ott et al. (2019b), or to estimate a $3 \mathrm{D}$ velocity field based on multiple parallel 2D velocity fields, synchronized with each other. The conditional approach shows good results when coupled with a phase-average process, acting as filtering Schaetther et al. (2002) Hardy et al. (2010) Ostermann et a.1 (2015).

Estimation methods are preferred when the temporal or spatial resolution of the experimental rough data needs to be improved. Stochastic estimations, such as LSE / QSE methods (linear / quadratic stochastic estimation) Hudy et al. (2006) can then be implemented. The purpose is to use a local time-resolved conditioner signal to estimate a velocity field Fadla et al. (2016) Chovet et al.
(2017). This method shows good results in a wide range of application (isotropic turbulence Adrian ( bulent boundary layers Guezennec and (hoil (11988), axisymmetric jets Bonnet et a.l. (11994), descending steps Cole and (Glawsen (ए998), open cavities Murray and Ukeiley (2003)...). Deep learning methods, based on the emerging field of machine learning Turing ([450), also begin to find their applications to flow field estimation, thanks to their ability to assimilate a large number of data and extract inter-correlations or paradigms. Supervised learning methods have shown their effectiveness in reconstructing $2 \mathrm{D}$ velocity and pressure fields around a cylinder, based on local pressure measurements Bright et al. (2013). More recently, neural network learning has been used to greatly improve the spatial and temporal resolution of low-resolution $2 \mathrm{D}$ velocity fields in the wake of a cylinder lin et al. (2018), Tin et al. (2020).

Indeed, this kind of deep learning tools have benefited large developments thanks, mainly, to computer capabilities in the past years while being known from decades. Sophisticated neural networks based on physicsconstrained deep learning strategies have been recently proposed: Raissi et al. (2019), Li and Allen-Zhul (2019), Sun et al. (2019), Sim et a. (2020). However large-area flow field measurements with both high spatial and temporal resolution remain a real challenge Lee and You (2019). These estimation methods have a common objective: to obtain space- and time-resolved flow field when only restricted data are available (low spatial or temporal resolution).

In this framework, a slotted pulsed jet blowing at 100 $\mathrm{Hz}$ with a peak velocity of $90 \mathrm{~m} / \mathrm{s}$ through a $0.5 \times$ $30 \mathrm{~mm}$ slot inclined at $45^{\circ}$ is used in order to test the present flow field estimation method. Previous studies (Ot.t $([20201))$ shown that particle seeding is not compatible with this configuration to characterize the flow dynamics, but $20 \mathrm{kHz}$ hot-wire measurements have been successfully conducted along a 3D space-resolved measurement grid. In order to obtain a 3D space-time-resolved velocity field based on these local measurements, we propose in the present study a Neural Network-based flow field estimation method able to give a 3D spacetime-resolved velocity field using local time-resolved velocity signals captured with a hot-wire traveling along a 3D measurement grid. To validate this method, the same experimental data set is processed using a previouslyvalidated conditional-based approach Ot.t et al. (2019b), which allows to obtain a 3D space-time-resolved baseline flow field.

The paper is outlined as follows. The experimental setup and the two estimation methods are detailed in section ㅁ. The neural network approach is validated in section 
[ 3 in two steps: firstly with a quantitative study of the learning and test losses for different sampling rates, and secondly with an in-depth comparison of the results obtained by the neural network with the results of the conditional approach. The use of neural network based methods as flow field estimation is discussed in section 田, and its benefits and perspectives are clarified. Section briefly concludes this study.

\section{Apparatus and estimation methodologies}

\subsection{Experimental setup}

The experimental setup used to capture the reference data set, consists in a $45^{\circ}$ inclined slotted pulsed jet, blowing in a confined space, without crossflow. The present experiment was performed in the ONERA-LMFL boundary layer wind tunnel, which has a cross-sectional area of $30 \times 30 \mathrm{~cm}^{2}$. The pulsed jet is generated by a Festo valve, with a working frequency of $100 \mathrm{~Hz}$ (using a duty-cycle of $50 \%$ ). An inlet pressure of 5 bars regulated with 0.01 bar accuracy is used (Centronics pressure valve). The outlet of the valve is connected to a diffuser and a nozzle. The goal is to obtain a homogeneous jet (in terms of velocity amplitude) at the outlet of the nozzle. The nozzle is $45^{\circ}$ inclined, and has a cross-sectional area of $30 \times 0.5 \mathrm{~mm}^{2}$. The simple hotwire is mounted on a traveling system, motorized with stepping motors enabling the hot-wire to move with an accuracy of $0.1 \mathrm{~mm}$ in all directions. DANTEC Dynamics single-hot-wire probe, type 55P15 (5 $\mathrm{\mu m}$ diameter and $1.25 \mathrm{~mm}$ long plated tungsten wire sensor), operating at constant temperature connected to a DANTEC Dynamics conditioner (featuring a Wheatstone bridge) are employed to acquire the velocity induced by the actuator at the nozzle outlet. The sensor is calibrated on a DANTEC calibration bench before each wind tunnel tests, and a verification calibration is performed after each test to monitor the polynomial calibration law evolution. The velocity measurements are sampled at $20 \mathrm{kHz}$ and are low pass filtered with a cutting frequency of $10 \mathrm{kHz}$. The test duration is chosen to be equal to $2 \mathrm{~s}$. The 5049 cells measurement grid has a size of $40 \mathrm{~mm}$ in the Z-direction, with a $5 \mathrm{~mm}$ resolution, and are centered with the nozzle. In the X-direction, the grid has a size of $80 \mathrm{~mm}$ with a $5 \mathrm{~mm}$ resolution and is placed so that the nozzle is centered at $\mathrm{X}=5 \mathrm{~mm}$ from the left border of the grid. In the Y-direction, the grid is 30 $\mathrm{mm}$ high and begins at a distance of $0.3 \mathrm{~mm}$ from the wall. The resolution of the measurement grid along the $\mathrm{Y}$-axis is variable (from $0.2 \mathrm{~mm}$ close to the wall, and up to $2 \mathrm{~mm}$ far from the wall). In this framework, for the results layout, a dimensionless orthonormal coordinate system is defined, whose origin is the center of the actuator slot. The dimensions along $\mathrm{X}$ - and $\mathrm{Y}$-axis are normalized by the slot width: $X^{d}=\frac{X}{d}$ and $Y^{d}=\frac{Y}{d}$ with $d=0.5 \mathrm{~mm}$. Dimensions along Z-axis are normalized by the slot length: $Z^{l}=\frac{Z}{l}$ with $l=30 \mathrm{~mm}$. The experimental setup is shown on Fig. $\square$. It allows to capture the local time-resolved velocity signals along the present volumic measurement grid. For each measurement, the signal used to control the actuator (square signal at $100 \mathrm{~Hz}$ with a DC of $50 \%$ ) is acquired simultaneously, and the rising edge of this square signal is used as a trigger for the synchronization.

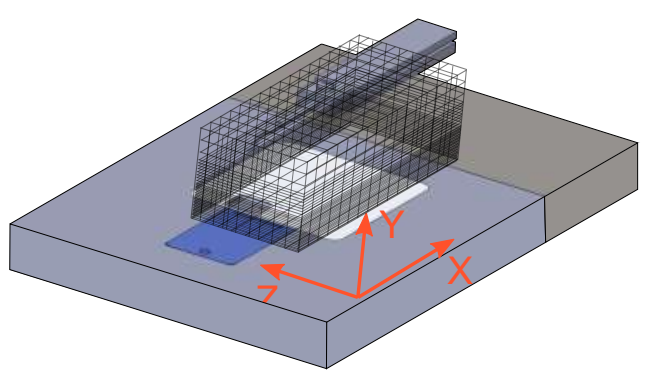

Fig. 1: Experimental setup and hot-wire measurement grid

\subsection{Estimation methodologies}

In this sub-section, we introduce the two different estimation methods used in the present paper. The goal of these methods is to express 3D time-resolved phaseaveraged velocity fields based on the local time-resolved velocity data-set obtained with the moving hot-wire. In the following sections, $U(t)$ denotes the rough velocity in physical time captured by the hot-wire sensor in $\mathrm{m} / \mathrm{s}$, $U_{\phi}\left(t^{*}\right)$ is the phase-averaged velocity in dimensionless time $\left(t^{*}=t / T\right.$ with $T$ the actuator period), and $U_{R M S}$ is the root mean square of the phase-averaged velocity. For a given spatial location $k_{a}$ (defined by a X,Y and $\mathrm{Z}$ ), and at a given dimensionless time $t_{a}^{*}$, the phaseaveraged velocity $U_{\phi}$ is defined by:

$U_{\phi}\left(k_{a} ; t_{a}^{*}\right)=\frac{1}{N} \sum_{i=0}^{N-1} U\left(k_{a} ; t_{a}^{*}+i T\right)$

and the phase-averaged root mean square velocity $U_{R M S}$ by:

$$
U_{R M S}=\sqrt{\frac{1}{N} \sum_{i=0}^{N-1}\left[\left(U\left(k_{a} ; t_{a}^{*}+i T\right)\right)^{2}-\left(U_{\phi}\left(k_{a} ; t_{a}^{*}\right)\right)^{2}\right]}
$$


where $T$ denotes the actuator period and $N$ is the number of periods used in the phase-average process.

\subsubsection{Conditional approach}

The conditional approach used in this paper is based on a method already used and validated on $2 \mathrm{D}$ cases Ott et al. (2019a) Chovet et al. (2016), and extrapolated to the 3D cases Ott et al. (2019b) Ott (2020). This approach is implemented through a Python program, and processed using eight core processors (10240 $\mathrm{Kb}$ each). The local hot-wire measurements acquired simultaneously with the synchronization signal are processed in four steps. The first step is the synchronization of all local time-resolved measurements with each other. The second step consists in performing a classical phase-average $U_{\phi}$ over these periods. The third step is the distribution of the data into a three-dimensions tensor, and the fourth and last step is the extraction of the instantaneous velocity snapshots for each time step. These four steps are described in detail in Appendix A1 and shown in Fig. $\mathbf{\text { . }}$.

\subsubsection{Neural network}

Neural networks are nonlinear regression tools that have extensively been used in image recognition and language processing Kotu and Deshpande (2019). According to the universal approximation theorem, any function can be approximated by a sufficiently large and deep network Hornik (ए99ा). In this paper, a neural network is used to express the complete phase-averaged velocity field as a nonlinear function of spatial coordinates and time. A general introduction to the neural network (including terminology, functioning of a neuron and a neural network), is detailed in Appendix A2.

In this paper, training data are phase-averaged local velocity signals based on hot-wire measurements and the objective is to learn optimal weights and biases to express the phase-averaged velocity field and turbulent statistics as a function of spatial coordinates and dimensionless time. In terms of neural network, input neurons are $X, Y, Z, t^{*}$ and output neurons are $U_{\phi}\left(X, Y, Z, t^{*}\right)$ and $U_{R M S}\left(X, Y, Z, t^{*}\right)$. Since the goal of this paper is to validate the use of a neural network for flow field estimation based on local hot-wire measurements, the neural network architecture is not subject to in-depth optimization. Goodfellow et al. (2015) showed that the architecture presented by Szegedy et al. (2014) (a three-layer 100-100-10 non-convolutional neural network with a Sigmoid activation function) showed good performance to limit the disturbances existing in the inputs data, in particular for the recognition of linearly noisy images. Furthermore, the work of Goodfellow et al. (2015) and Szegedy et al. (2014) underlines the fact that, in adversarial examples, the higher layers are significantly more useful than those on the lower layers. Following these recommendations and a preliminary trial-error study, the chosen architecture is composed of three hidden layers with respectively 15, 11 and 9 neurons, which allowed to limit the impact of the noise present in the hot-wire measurements used for the training of the neuron network, and thus obtain a more robust estimation. The cost function considers all hotwire locations (superscript $i$ ) and all samples (index $t$ ), yielding:

$E_{2}=\sum_{t, i}\left\|\mathbf{U}_{\phi_{t}}^{(i)}-\mathrm{NN}_{t}^{(i)}\right\|^{2}$

Where $\mathbf{U}_{\phi_{t^{*}}}^{(i)}$ is a vector containing $U_{\phi}\left(X_{i}, Y_{i}, Z_{i}, t^{*}\right)$ and $U_{R M S}\left(X_{i}, Y_{i}, Z_{i}, t^{*}\right)$ while $\mathrm{NN}_{t}^{(i)}$ contains the neural network estimate. The gradient descent (evaluated with batches of 1000 samples) is done over 1500 epochs (one epoch beeing one pass over the entire dataset). In order to make sure that the neural network training is converged and not over-fitted after these 1500 epochs, the cost function is monitored for the crossvalidation data and the training data. In this framework, the cross-validation/training ratio is set to $10 \%$. The neural network was implemented under Python, using the Keras library Keras (2018). The final training procedure is summarized in Fig.ㄱ. Finally, the neural network acts as an optimal nonlinear interpolator. Naturally, the success of such estimation depends on the training data quality (hot-wire locations Lakshminarayanan et al. (2016)), the good choice of hyperparameters and the learning procedure.

\section{Results}

The experimental setup presented in $[2]$ is used to capture the hot-wire database and the space- and timeresolved velocity fields are reconstructed using the $3 \mathrm{D}$ conditional approach (2.2.]) and the deep learning approach ( 2.2 .2$)$. However, the raw measurements represent a very large database: 5049 measurement locations, 2 seconds of measurement time for each point with a frequency of $20 \mathrm{kHz}$, which represents a total of $20 \times 10^{7}$ samples. As the neural network structure is chosen in this study to be more versatile and able to be used in a large number of cases and with noisy signals, the learning duration of such a database is estimated at more than 30 hours with the hardware configuration used in 
Experimental hot-wire measurements

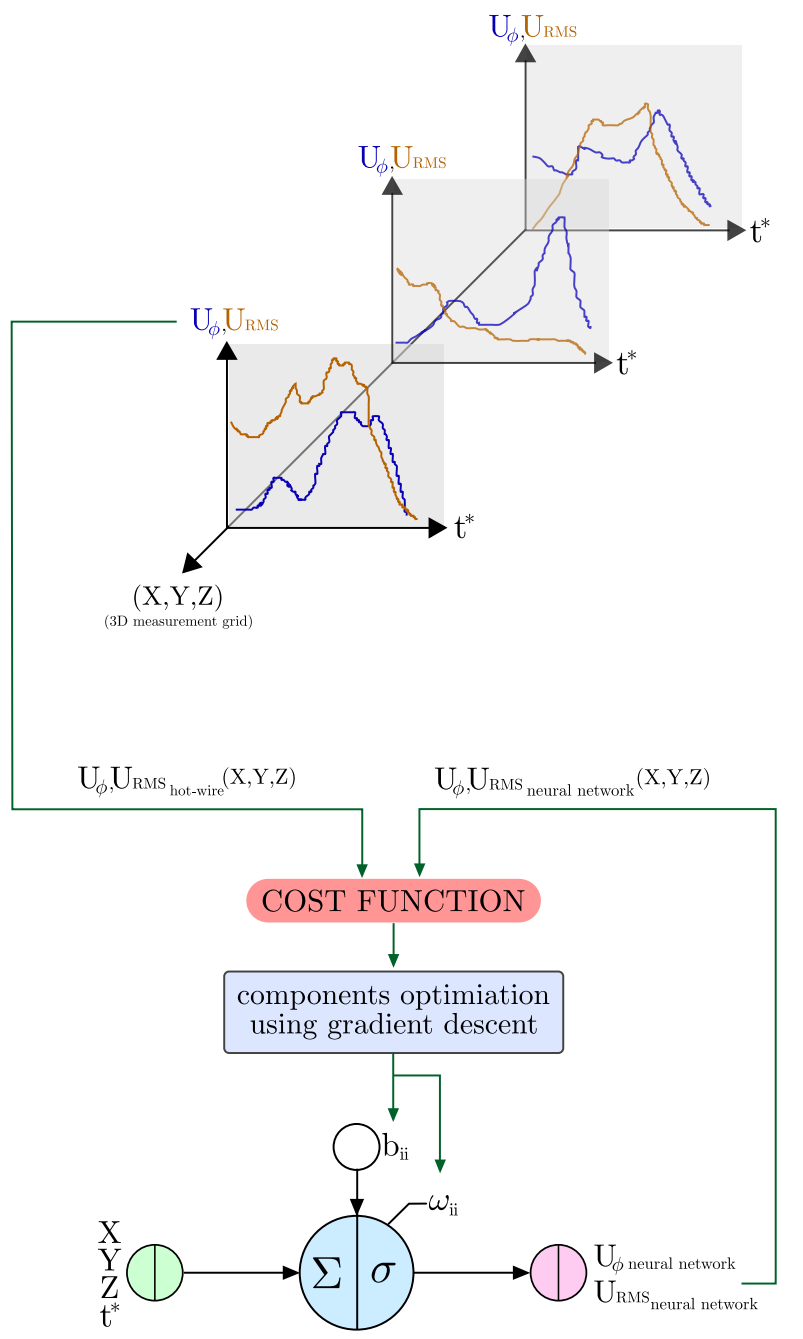

Neural network $[4 \mathrm{i} ; 15 ; 11 ; 9 ; 2 \mathrm{o}]$

Fig. 2: Neural network training

this study (8 core CPU with 8 GB of RAM). In comparison, processing the same database using the conditional approach takes 25 minutes. To overcome this high computational cost, a reduction in the quantity of data used for training the neural network is carried out. For this reason, the present section is divided into two sub-section. The first one aims at investigating the effects of the amount of data used for training the neural network on the quality of the results, and select a satisfactory case. The chosen case is then subject to an in-depth study in the second sub-section.

\subsection{Neural network's reliability study}

In this sub-section, the neural network's architecture is tested for different amounts of training data in a range of $10 \%$ to $30 \%$ (with a $5 \%$ increment) of the raw database (corresponding respectively to $10.0 \times 10^{4}$ and $30.3 \times 10^{4}$ samples). The data are randomly selected over the raw database $\left(10.0 \times 10^{5}\right.$ samples $)$, and used as input for the neural network. The results of these data processing are shown on Fig. 3 .

In order to quantify the network's reliability, three tools are used (corresponding to the three columns of the Fig. 3). The first one is the monitoring of the learning losses over epochs. It consists in the calculation of the cost-function (MSE) at each epoch over the training set (orange line) and over the test set (dashed green). The second tool are the estimated flow fields of $U_{\phi}$ and $U_{R M S}$ that can be compared to the reference base flow obtained with the conditional approach. It allows to check the flow field estimations relevance and to visualize the smoothing in space. For these visualizations, a two-dimensions $\mathrm{Z}$ plan is extracted from the volume estimations, at the middle of the slot $\left(Z^{l}=0\right)$ for two representative instants $\left(t^{*}=0.1\right.$ and $\left.t^{*}=0.25\right)$. On these snapshots, the phase-averaged velocity is colored using the dark end rainbow color scale, and the RMS velocity is colored using the blue-red color scale. The third tool is a local time-resolved plotting of the neural network phase-averaged velocity estimation at a strategic location. The location is chosen close to the actuator where the velocity gradient is the highest, at $X^{d}=30, Y^{d}=20$ and $Z^{l}=0$ (mid-plan, $15 \mathrm{~mm}$ downstream the slot and $10 \mathrm{~mm}$ from the wall). The reference phase-averaged velocity obtained with the conditional approach is plotted in dark blue over dimensionless time $t^{*}$, and the phaseaveraged velocity estimated with the neural network is plotted in light blue over dimensionless time $t^{*}$.

The local time-resolved phase-averaged velocity signal plotted on Fig. B show that the amount of data used in the training process of the neural network has a minor effect on the extremum amplitudes: the velocity peak amplitude are well estimated in every case. However, the temporal reliability and the global shape of the estimated signal (particularly the width of the blowing peak) are drastically improved when the amount of data used increases: the quadratic error between the neural network estimation and the base flow at this specific location decreases from 1.3 at $10 \%$ to 0.6 at $30 \%$. The same observation can be done on the $2 \mathrm{D}$-flow fields on both instants chosen: when the amount of data used is low, the jet envelop is smoothed and can be overestimated at some locations in terms of phase-averaged velocity, and is globally smoothed and underestimated 
1

2

3

4

5

6

7

8

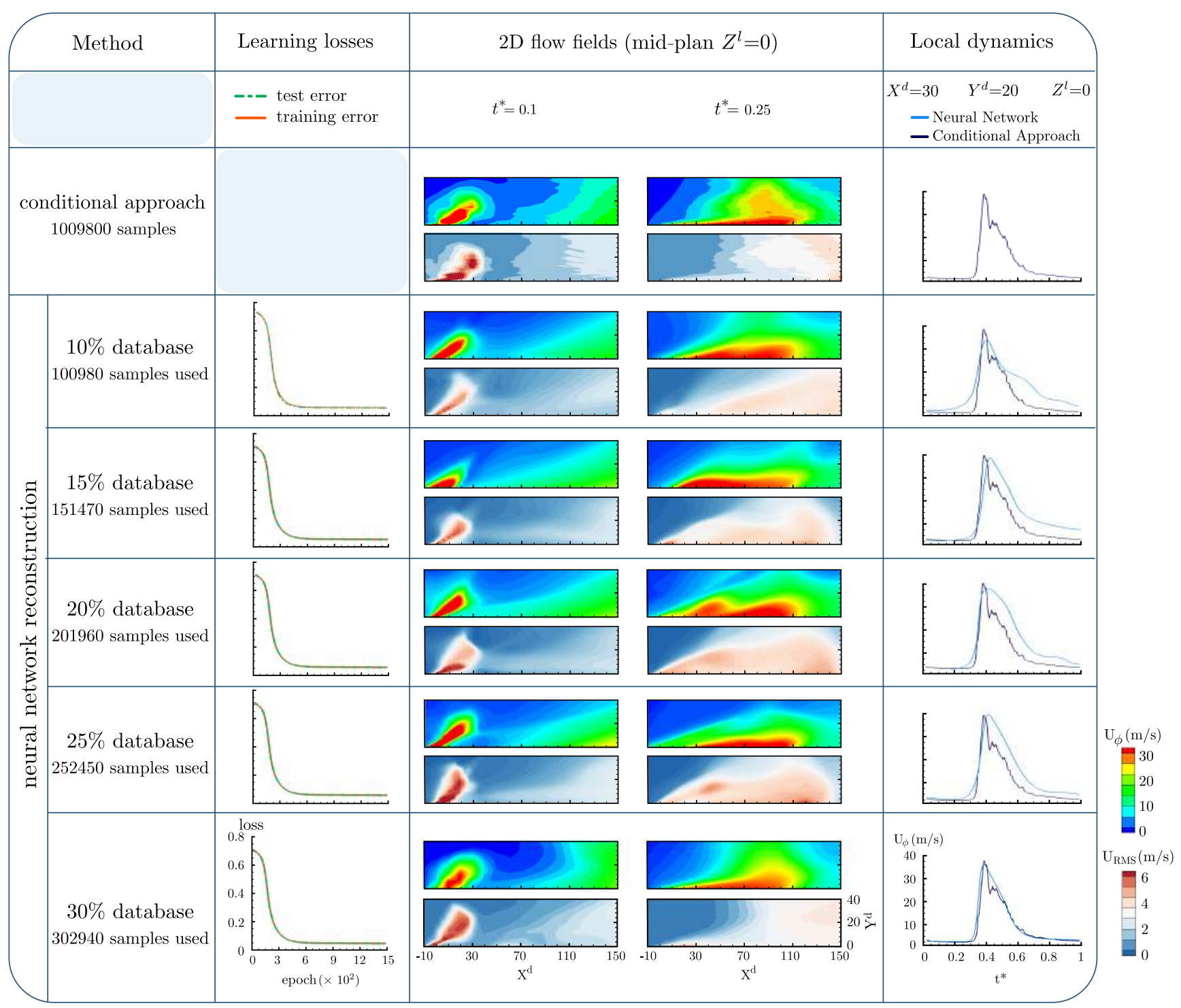

Fig. 3: Effect of the data training amount on the accuracy of the phase-averaged flow field estimation

in terms of RMS velocity. The reliability of these estimations increases with the amount of data used. Learning losses quantification is a really interesting tool to quantify the neural network capability, and monitor the training process over epochs. With $10 \%$ of the database, the training error is higher than the test error for the first tens epochs, which means that at this stage the neural network is more efficient to estimate the data already processed than the data set aside for the test. The loss function is at 0.73 at the end of the first epoch for the test error, and 0.71 for the training error. The losses quickly decrease to respectively 0.035 and 0.034 at 600 epochs, and decrease slowly to end up at a plateau of respectively 0.031 and 0.030 from 900 epochs, meaning that the weights and bias of the neural network are converged. Furthermore, the losses curves are superim- posed, which means that there is no over-fitting in this case. The losses curves behave similarly for all percent of data used, except that when the amount of data used increases, the difference between the test and the training errors at the first epoch decreases. The values of the loss function after the convergence of the losses are however almost similar for each case $(0.03 \pm 0.001)$.

Particular attention must be paid to the fact that this quadratic error is calculated with respect to the data set used as input to the neural network for each case. These quantities therefore can not be compared with each other since the input data set is not the same. In order to quantify and compare the reliability of each model, it is mandatory to evaluate the cost function of each model with respect to the original $10 \times 10^{5}$ samples database. This evaluation is therefore performed 
over all the models presented in Fig. [1, regarding $U_{\phi}$. The quadratic errors of the $U_{\phi}$ estimation using the different percentages of training data are then compared on Fig. 四.

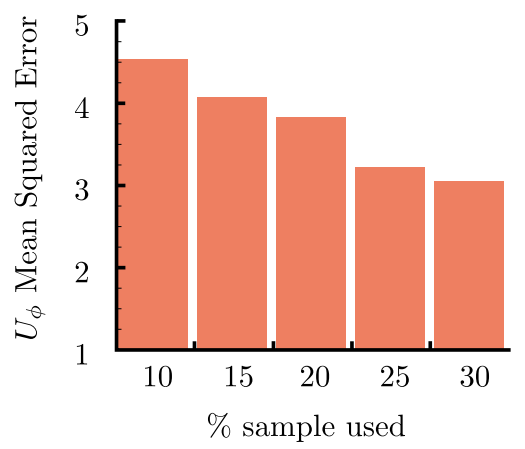

Fig. 4: Quadratic error

This comparison shows that by expanding the input database from $10 \%$ to $30 \%$, the MSE (mean squared error) of the $U_{\phi}$ estimation can be lowered by $39 \%$ (from an MSE of 4.5 to 2.7). Thus the estimated flow field is more reliable when $30 \%$ of the database is used as input data for the neural network, but the computing time is also higher.

The $30 \%$ case showing good reliability in terms of quadratic error on $U_{\phi}$ and good training convergence, it is selected as the best reliability/calculation cost compromise in this framework. It is therefore used in the following subsection to carry out the in-depth study of the flow field estimations and a detailed comparison with the reference base flow obtained with the conditional approach.

\subsection{In-depth study of the results}

This sub-section aims to compare the 3D fields obtained with the conditional approach and the neural network using randomly $30 \%$ of the database. The reconstructed velocity-fields using the conditional approach are plotted on Fig. (1 (a) (b) and (c), corresponding to three different dimensionless time instants $t^{*}$. The same phaseaveraged velocity-fields are estimated using the neural network approach on Fig. 回 (d) (e) and (f). On these $3 \mathrm{D}$ flow fields, iso-velocity surfaces of $U_{\phi}=15 \mathrm{~m} / \mathrm{s}$ are plotted and colored by $U_{R M S}(\mathrm{~m} / \mathrm{s})$ using the blue-red color scale. The domain borders are colored by $U_{\phi}(\mathrm{m} / \mathrm{s})$ using the dark end rainbow color scale. 2D slices are extracted from the $3 \mathrm{D}$ reconstructed fields at $Z=20 \mathrm{~mm}$. On those proposed planes, the velocity fields are colored by $U_{\phi}(\mathrm{m} / \mathrm{s})$ using the dark end rainbow color scale, and the associated RMS fields are colored by blue-red color scale (g), (h), (i) for the conditional approach and (j), (k), (1) for the neural network approach). The spatial development of the jet reconstituted using the 3D conditional approach is considered as the reference flow thanks to its validation in Ott et al. (2019b) and using comparisons with literature about slotted and pulsed jet development Eroglu and Breidenthal (2000), Vernet et all (2009), Mahesh (2013), Sau and Mahesh (2010). The Fig. 回 gives the results of the conditional approach (left) and those related to the neural network (right). The 3D jet dynamic development corresponds, as expected, to those observed in the literature. The jet is symmetrical with regard to the plane $Z^{l}=0$ (mid-slot, with $Z^{l}$ defined by $Z / l$ with $l$ the slot length). The three-dimensional effects of the jet are correctly captured and reconstituted here. The flow features are coherent and continuous in space and time. At the instant $t^{*}=0.1$ ( $2 \mathrm{~ms}$ after the valve opening), the blowing front coming out of the slot can be discerned. The jet is homogeneous along the slot, and despite the $45^{\circ}$ inclined nozzle, it remains confined close to the wall, as shown on Fig. (a) (a) and (d). At this instant, the jet only modifies a reduced area ( $1 l$ wide, $0.5 l$ long and $0.1 l$ thick, with $l$ the length of the slot). The slot edges $\left(Z^{l}= \pm 0.5\right)$ are the first areas where structures are produced, inducing two velocity peaks. These peaks are enclosed in cylindrical structures parallel to each other and aligned with $Z^{l}=-0.5$ and $Z^{l}=+0.5$. These structures correspond to the formation of a pair of counter-rotating vortices, which are well identifiable on plot (a). The corresponding structures obtained by the neural network on plot (d) can be perceived despite a pronounced smoothing of the iso-surfaces shape. These two vortices then loose their intensity to form a homogeneous blowing front along the Z-axis. From $t^{*}=0.25$ (h) for the conditional approach and (e) for the neural network), a shear layer vortex created at the leading edge of the slot is also formed above the jet core, slightly upstream the blowing front. This structure is centered on $X^{d}=34$ at $t^{*}=0.25$ and on $X^{d}=82$ at $t^{*}=0.75$ (on plot (C) and (f)). At the instant $t^{*}=0.75$ ( $5 \mathrm{~ms}$ after the valve closing), it is noticeable that the blowing extinction is not homogeneous. The jet first stops blowing in the middle of the slot but remains at $Z^{l}=-0.5$ and $Z^{l}=+0.5$. Thus the two contra-rotating vortices continue to be fed, which induce the generation of conical structures centered on $Z^{l}=-0.5$ and $Z^{l}=+0.5$ for $X^{d} \in[0 ; 70]$. All these topological elements observed in the reference velocity field (obtained with the conditional approach on plot (a), b) and (C) and in the literature are well estimated using the neural network approach, while ensuring data continuity as shown by the $3 \mathrm{D}$ reconstructed fields on plot (d), (e) and (f). The velocity fields extracted from the 3D fields at mid-plane perpendicularly to the slot and the 
wall also show good restitution of the phase-averaged dynamic response of the actuator inside the jet compared to the base flow. Plot (g), (h) and (i) show that the jet core (defined by $U_{\phi} \geq 30 \mathrm{~m} / \mathrm{s}$ ) is $45^{\circ}$ inclined at $t^{*}=0.1$, with a jet core length of $36 d$ (with $d$ the slot width). The jet is then confined close to the wall and is $120 d$ long at $t^{*}=0.25$ (on (h)).

At this time the shear layer vortex is clearly visible. During the blowing half-period, the average core velocity is $U_{\phi}=32 \mathrm{~m} / \mathrm{s}$, with an average RMS velocity of $U_{R M S}=7 \mathrm{~m} / \mathrm{s}$. At $t^{*}=0.75$ (i)), the actuator is turned off, and the last flow features are advected (mainly the jet core and the shear layer vortex). Plot (j), k and (1) show the flow-fields obtained using the neural network approach. At $t^{*}=0.1$ (j), the jet core is $45^{\circ}$ as expected given the previous results. However, the jet core is only $0.4 \mathrm{~L}$ long, and the average RMS velocity inside the jet core is $U_{R M S}=5 \mathrm{~m} / \mathrm{s}$. At $t^{*}=0.25(\mathrm{k})$, the mean and RMS velocities fields are smoothed (jet core is only $1.5 \mathrm{~L}$ long) and the shear layer vortex is observable but its boundary is not sharply demarcated. The advection velocity of the main flow features is well modeled (the vortex and jet core are located where they are expected with regard to the conditional approach). At $t^{*}=0.75$ (1) the jet envelop is well estimated despite a slightly smoothed border. The RMS field also show a globally underestimated intensity without modifying the shape of the RMS field and isolines. Overall the comparison of $2 \mathrm{D}$ fields allows to highlight that the standard deviations seem to be globally underestimated by the neural network: on average by $36 \%$ in the jet core, and by $12 \%$ in its surrounding. Moreover, the outline of the jet core is smoothed and spread out: the jet core surface and the jet length are underestimated. However, the mesh of the neural network estimation plot is not degraded compared to the base flow plot one, which means that the method allows to obtain the same overall phase-averaged flow dynamics as in the base flow by using only $30 \%$ of the experimental data. In order to quantitatively study the performance of the neural network method, the signals reconstructed by this method are extracted locally for a phase-averaged period and overlaid with the phase-averaged hot wire experimental data. These comparisons are shown in Fig. [6. The comparison locations are chosen close to the actuator slot, where the velocity gradient is the highest, and thus where the velocity estimation in time is more challenging. These locations are the following: the point (1) is placed at $X^{d}=2, Y^{d}=1$ and $Z^{l}=0(1 \mathrm{~mm}$ downstream the slot and $0.5 \mathrm{~mm}$ from the wall). The point (2) is at $X^{d}=30, Y^{d}=20$ and $Z^{l}=0(15 \mathrm{~mm}$ downstream the slot and $10 \mathrm{~mm}$ from the wall). Fig. (1) and (2) show that the main dynamic of the signal is well estimated by the neural network. Indeed, plot (1) shows that the blowing edge reach this location at $t^{*}=0.15$ on the reference base flow (Conditional Approach), and at $t^{*}=0.17$ on the signal given by the neural network, i.e. an error of $13 \%$. The velocity peak occurs at $t^{*}=0.31$ for the reference signal and is anticipated by the neural network at $t^{*}=0.30$ (3\% error). On plot (2), this error is reduced to $6 \%$ for the blowing edge $\left(t^{*}=0.31\right.$ for the reference signal estimated at $t^{*}=0.29$ by the neural network) and to $2 \%$ for the velocity peak $\left(t^{*}=0.38\right.$ for the reference signal and $t^{*}=0.37$ for the neural network).

The dynamics and the average characteristics of the flow are locally well estimated by the neural network (the shapes of the curves are identical and the temporal errors remain low). However, the neural network strongly smooths the velocity variations of low amplitude, filtering out high frequencies.

In terms of amplitude the time-averaged velocity is well estimated by the neural network, with an underestimation of $5 \%$ at the location (1) and overestimation of $12 \%$ at the location (2). However, the velocity peaks are both underestimated by the neural network in terms of amplitude, with an error of $13 \%$ at (1) and $4.8 \%$ at (2).

This local comparison confirms the observations made on $2 \mathrm{D}$ and $3 \mathrm{D}$ fields: the neural network is able, using only $30 \%$ of the original data, to reliably estimate the phase-averaged velocity, especially the mean behavior of the velocity. However, the velocity signals are smoothed (high-frequency features are filtered) and tend to underestimate the velocity amplitude.

These limitations of the neural network-based method (underestimated velocity intensity and smoothed outline of the jet core) could result from various roots:

○ the removal of $30 \%$ of the database

○ the neural network architecture

$\circ$ a too small number of trained weights in the neural network

○ the choice of the activation function in the neural network

The method however allows a reliable estimation, since the main flow features are well estimated in space and time. The reconstruction by the neural network is therefore a relevant method for the $3 \mathrm{D}$ estimation of space-time-resolved fields, based on the learning of experimental data chosen randomly in space and time.

\section{Global discussion}

This section aims to compare the effectiveness and capabilities of both methods presented in this paper. Table $\mathbf{m}$ gathers the advantages and limitations found in 
1

2

3

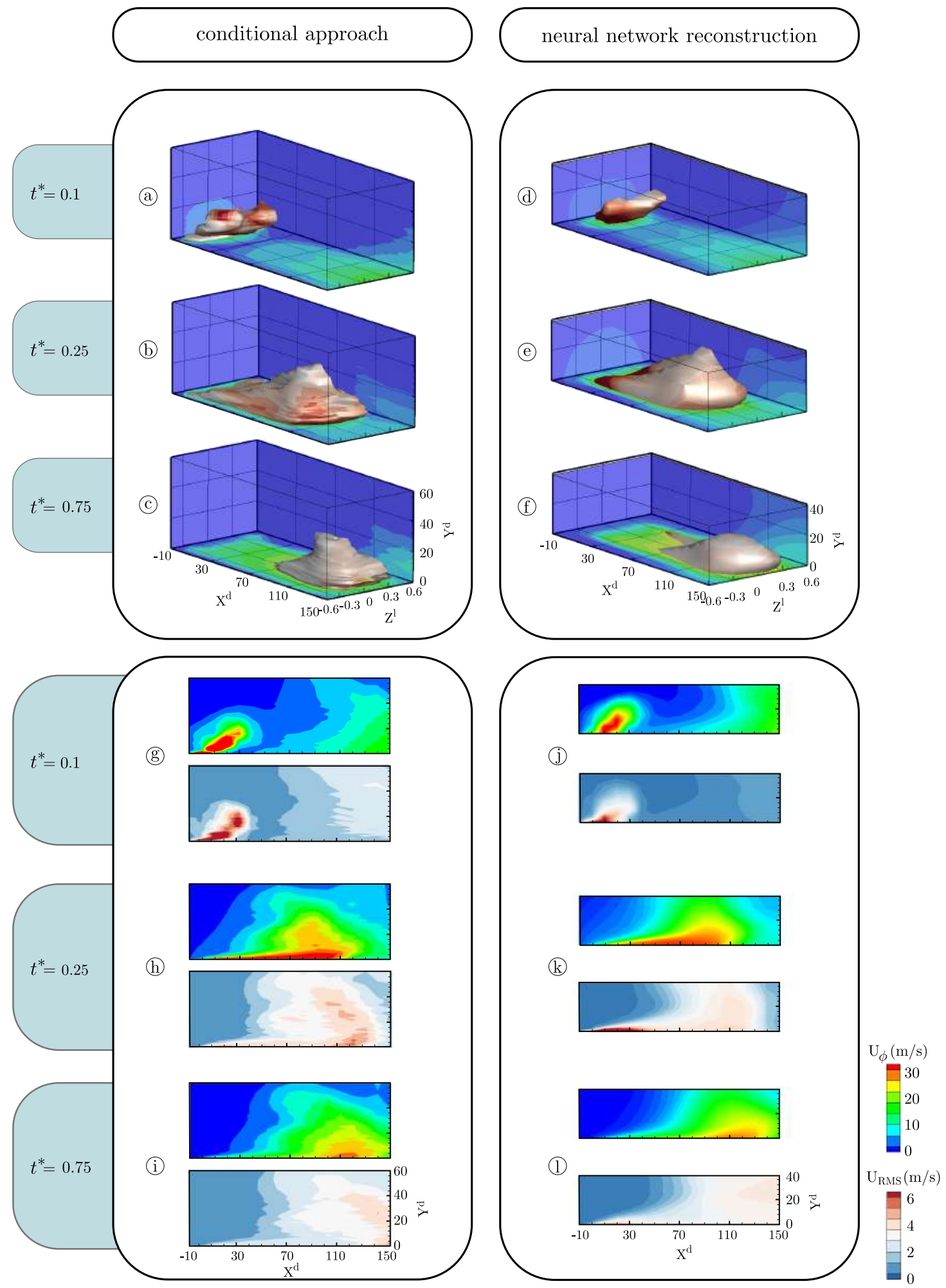

Fig. 5: Results and methods comparison 


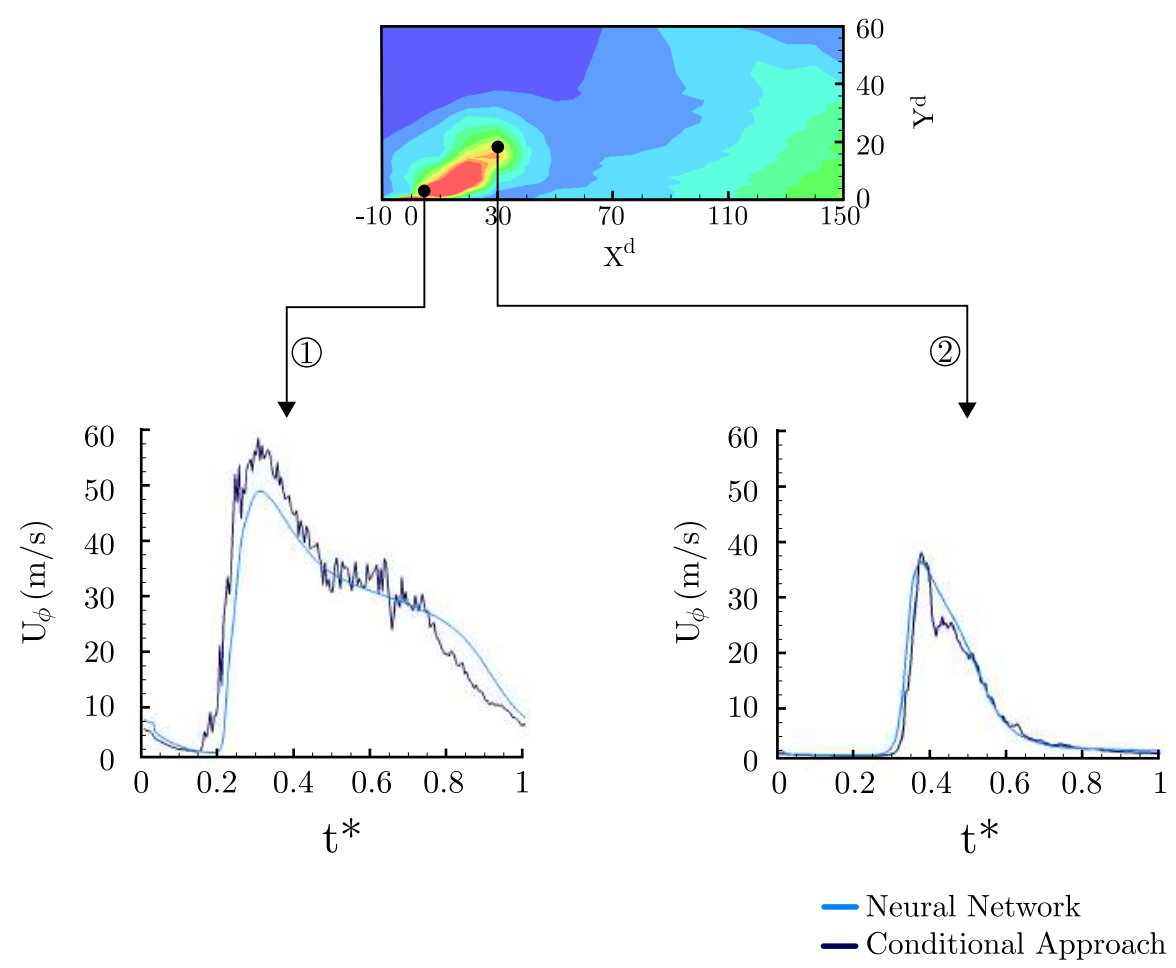

Fig. 6: Local estimation in time

the present study regarding the use of the neural network versus the conditional approaches. The conditional approach is a versatile and quick method that is simple to implement. The neural network, meanwhile, requires more time and preliminary studies to be implemented and validated. Increased attention has to be brought to the analysis of the results in order to validate the consistency of the estimated physics. The conditional approach is quicker than the present neural network in terms of computational time. The complete process (from rough data to reconstructed flow fields) is 70 times higher using the neural network than the conditional approach (30 hours versus 25 minutes). The learning process of the neural network itself is responsible for $99.5 \%$ of the computational time, which means that once the model is defined the time needed for the flow field estimation is neglectable. Furthermore, the conditional approach only provides flow fields with a coarser or equal resolution (in space and in time) than the experimental data, while the neural network approach allows refining both spatial and time-resolutions. This comes from the fact that the conditional approach only reconstitutes the flow field based on the experimental measurements, while the neural network can model the complete flow field. This study has shown that it is possible to improve drastically the temporal and spatial resolution of the flow field estimation compared to the resolution of the measurements, without too much flow dynamics losses. The main limitation of the present neural network approach is the processing time, which led in this paper to a randomized reduction of the learning database and therefore a deterioration of the estimation. Furthermore, the neural network method showed smoothed velocity fields and underestimated standard deviations, which could come from the fact that only $30 \%$ of the original data were used, or that the architecture of the neural network was not perfectly suited (too low number of weights, activation function choice ...). In order to decrease the computational time of the learning process while increasing the input database size, it would be necessary to look to structural optimization of the neural network and its architecture, as proposed by Dubois et all (20201) and Idrissi et all (2016). Indeed, a too-small number of trained weights (i.e. not enough hidden layers/neurons) can lead to smoothed results, while too many trained weights (i.e. large number of hidden layers/neurons) can lead to over-fitted results. Parameter studies can provide a more robust neural network with better performances (Hamwood et al (2018)). In addition, rather than using the classical sigmoid activation function, the literature suggests that using more subtle activation function (e.g. ReLUs functions) could allows to prevent neural network overfittings (Ramachandran et al] (20118)) .

Such an optimized neural network could open doors to many interesting applications in the flow field esti- 
1

2

\begin{tabular}{lll} 
Approach & advantages & limitations \\
\hline & $\circ$ spatial and temporal resolution enhancement & \\
& $\circ$ quick reconstruction & \\
Neural Network & $\circ$ great development potential & $\circ$ implementation complexity \\
& $\circ$ nonlinear modeling & \\
& $\circ$ flexibility & \\
& $\circ$ light implementation & restricted spatial and temporal resolution \\
Conditional & $\circ$ quick process & mation a full process for each flow field esti- \\
& $\circ$ robust method & $\circ$ absence of modeling
\end{tabular}

Table 1: Advantages and limitations of the estimation approaches

mation domain. It could be, for instance, theoretically possible to enhance drastically the estimated area or to investigate boundary conditions that have not been investigated experimentally. This could be achieved for instance by adding the actuation frequency in the input data, and by training the network with random measurements (different positions, instants, and actuation frequencies). It might be then possible to reconstruct the fields for several frequencies, even if these have not been investigated experimentally. Particular attention should then be paid to the distribution of the database used for training, as well as its resolution.

\section{Conclusion}

This study proposes a data processing approach to obtain a space-time-resolved velocity field at the exit nozzle of a slotted pulsed jet based on hot-wire local timeresolved velocity data. This method uses the periodic behavior of the flow and the actuator's command signal as a synchronization trigger. It is based on a neuronal network whose entries are the location and the time, and outputs are the mean and RMS velocities. This space-time-resolved estimation approach is validated by comparison with the conditional approach (previously validated) performed on the same experimental data set. The neural network approach shows good results in the phase-averaged dynamic restitution of the actuator's behavior, even by degrading the learning database (only $30 \%$ of the data are used), which means that this technique can be used to drastically improve the temporal or spatial resolution of a flow field estimation compared to the experimental data resolution. The main coherent structures are well captured and the flow behavior and physics are well estimated. The RMS velocity seems however to be underestimated by the neural network and the jet core is smoothed and spread out, due to the intentional degradation of the learning database.
There is no claim of optimality in the network architecture used in this framework, since it is based on the literature without further optimizations. However, the chosen architecture has proven to be robust for the interpolation task and may therefore be considered as a first trial of hyperparameters when post-processing large hot-wire signals database.

Furthermore, without any structural optimization of the neural network, the computational time required to obtain the flow field (which corresponds to the cumulative time of the learning process and the estimation), is about 70 times longer than the conditional approach for the same experimental database size. Since the flow field estimation processing time is about 600 times smaller than the learning time for the neural network method, in the case of single database studies requiring multiple reconstructions, the use neural network method can be of great benefit and save considerable time.

Acknowledgements This work was carried out within the framework of the CNRS Research Federation on Ground Transports and Mobility, in articulation with the ELSAT2020 project supported by the European Community, the French Ministry of Higher Education and Research, and the Hauts-de-France Regional Council. The authors gratefully acknowledge the support of these institutions. 


\section{References}

Adrian RJ (1979) Conditional eddies in isotropic turbulence. Physics of Fluids 22(11):2065-2070

Aeschlimann V, Barre S, Djeridi H (2013) Unsteady Cavitation Analysis Using Phase Averaging and Conditional Approaches in a 2D Venturi Flow. Open Journal of Fluid Dynamics 03(03):171-183

Béra JC, Michard M, Grosjean N, Comte-Bellot G (2001) Flow analysis of two-dimensional pulsed jets by particle image velocimetry. Experiments in Fluids 31(5):519-532

Bisgaard C (1983) Velocity fields around spheres and bubbles investigated by laser-doppler anemometry. Journal of Non-Newtonian Fluid Mechanics 12(3):283-302

Bonnet JP, Cole DR, Delville J, Glauser MN, Ukeiley LS (1994) Stochastic estimation and proper orthogonal decomposition: Complementary techniques for identifying structure. Experiments in Fluids 17(5):307-314

Bright I, Lin G, Kutz JN (2013) Compressive sensing based machine learning strategy for characterizing the flow around a cylinder with limited pressure measurements. Physics of Fluids 25(12)

Callaham JL, Maeda K, Brunton SL (2019) Robust flow reconstruction from limited measurements via sparse representation. Physical Review Fluids 4(10)

Cambonie T, Aider JL (2014) Transition scenario of the round jet in crossflow topology at low velocity ratios. Physics of Fluids 26(8)

Cambonie T, Gautier N, Aider JL (2013) Experimental study of counter-rotating vortex pair trajectories induced by a round jet in cross-flow at low velocity ratios. Experiments in Fluids 54(3)

Chovet C, Lippert M, Keirsbulck L, Foucaut JM (2016) Dynamic characterization of piezoelectric microblowers for separation flow control. Sensors and Actuators, A: Physical 249:122-130

Chovet C, Lippert M, Foucaut JM, Keirsbulck L (2017) Dynamical aspects of a backward-facing step flow at large Reynolds numbers. Experiments in Fluids $58(11)$

Cole DR, Glausen MN (1998) Applications of stochastic estimation in the axisymmetric sudden expansion. Physics of Fluids 10(11):2941-2949

Dubois P, Gomez T, Planckaert L, Perret L (2020) Data-driven predictions of the Lorenz system. Physica D: Nonlinear Phenomena 408

Emerick TM, Ali MY, Foster CH, Alvi FS, Popkin SH, Cybyk BZ (2012) SparkJet actuator characterization in supersonic crossflow. 6th AIAA Flow Control Conference 2012
Eroglu A, Breidenthal RE (2001) Structure, penetration, and mixing of pulsed jets in crossflow. AIAA journal 39(3):417-423

Fadla F, Graziani A, Kerherve F, Mathis R, Lippert M, Uystepruyst D, Keirsbulck L (2016) Electrochemical Measurements for Real-Time Stochastic Reconstruction of Large-Scale Dynamics of a Separated Flow. Journal of Fluids Engineering, Transactions of the ASME 138(12)

Fernandez P, Delva J, Ott C, Maier P, Gallas Q (2018) Experimental Measurement Benchmark for Compressible Fluidic Unsteady Jet. Actuators 7(3):58

Foucaut JM, Coudert S, Stanislas M (2009) Unsteady characteristics of near-wall turbulence using high repetition stereoscopic particle image velocimetry (PIV). Measurement Science and Technology 20(7)

Goodfellow IJ, Shlens J, Szegedy C (2015) Explaining and Harnessing Adversarial Examples. 3rd International Conference on Learning Representations

Guezennec YG, Choi WC (1988) Stochastic estimation of coherent structures in turbulent boundary layers. Proceedings of the International Centre for Heat and Mass Transfer pp 453-468

Haack SJ, Land HB, Cybyk B, Ko HS, Katz J (2008) Characterization of a high-speed flow control actuator using digital speckle tomography and PIV. 4th AIAA Flow Control Conference

Hamwood J, Alonso-Caneiro D, Read SA, Vincent SJ, Collins MJ (2018) Effect of patch size and network architecture on a convolutional neural network approach for automatic segmentation of OCT retinal layers. Biomedical Optics Express 9(7):3049

Hardy P, Barricau P, Belinger A, Caruana D, Cambronne JP, Gleyzes C (2010) Plasma synthetic jet for flow control. 40th AIAA Fluid Dynamics Conference

Hornik K (1991) Approximation capabilities of multilayer feedforward networks. Neural Networks 4(2):251-257

Hudy LM, Naguib A, Humphreys WM (2006) Stochastic estimation of a separated-flow field using wallpressure-array measurements. Collection of Technical Papers - 44th AIAA Aerospace Sciences Meeting 18:13520-13541

Idrissi MAJ, Ramchoun H, Ghanou Y, Ettaouil M (2016) Genetic algorithm for neural network architecture optimization. Proceedings of the 3rd IEEE International Conference on Logistics Operations Management, GOL 2016

Jin X, Cheng P, Chen WL, Li H (2018) Prediction model of velocity field around circular cylinder over various Reynolds numbers by fusion convolutional neural networks based on pressure on the cylinder. Physics of Fluids 30(4) 
Jin X, Laima S, Chen WL, Li H (2020) Time-resolved reconstruction of flow field around a circular cylinder by recurrent neural networks based on non-timeresolved particle image velocimetry measurements. Experiments in Fluids 61(4)

Keras (2018) Guide to the Sequential model - Keras Documentation

Kotu V, Deshpande B (2019) Deep Learning. Data Science 521:307-342

Kovasznay LSG (1949) Hot-wire investigation of the wake behind cylinders at low Reynolds numbers. Proceedings of the Royal Society of London Series A Mathematical and Physical Sciences 198(1053):174190

Lakshminarayanan B, Pritzel A, Blundell C (2016) Simple and Scalable Predictive Uncertainty Estimation using Deep Ensembles. Cornell University

Lee S, You D (2019) Data-driven prediction of unsteady flow over a circular cylinder using deep learning. Journal of Fluid Mechanics 879:217-254

Li Y, Allen-Zhu Z (2019) What can resnet learn efficiently, going beyond kernels? In: 33rd Conference on Neural Information Processing Systems

Li Y, Chang J, Kong C, Wang Z (2020) Flow field reconstruction and prediction of the supersonic cascade channel based on a symmetry neural network under complex and variable conditions. AIP Advances 10(6)

Mahesh K (2013) The Interaction of Jets with Crossflow. Annual Review of Fluid Mechanics 45(1):379407

Murray NE, Ukeiley LS (2003) Estimation of the flowfield from surface pressure measurements in an open cavity. AIAA Journal 41(5):969-972

Olchewsky F, Desse JM, Donjat D, Champagnat F (2019) Vertical digital holographic bench for underexpanded jet gas density reconstruction. Digital Holography and 3D Imaging $\mathrm{p}$ Th2B.3

Ostermann F, Woszidlo R, Nayeri CN, Paschereit CO (2015) Phase-Averaging Methods for the Natural Flowfield of a Fluidic Oscillator. AIAA Journal 53(8):2359-2368

Ostermann F, Godbersen P, Woszidlo R, Nayeri CN, Paschereit CO (2017) Sweeping jet from a fluidic oscillator in crossflow. Physical Review Fluids $2(9): 2,90512$

Ott C (2020) Space-time resolved fluidic actuators characterization, and experimental identification of the physical mechanisms involved in their interaction with a boundary layer. $\mathrm{PhD}$ Thesis

Ott C, Gallas Q, Delva J, Lippert M, Keirsbulck L (2019a) High frequency characterization of a sweeping jet actuator. Sensors and Actuators, A: Physical 291:39-47
Ott C, Gallas Q, Delva J, Lippert M, Keirsbulck L (2019b) Interaction between a jet and a turbulent boundary layer. In: AIAA AVIATION Forum, Dallas, USA

Poelma C, Mari JM, Foin N, Tang MX, Krams R, Caro CG, Weinberg PD, Westerweel J (2011) 3D Flow reconstruction using ultrasound PIV. Experiments in Fluids 50(4):777-785

Raissi M, Perdikaris P, Karniadakis GE (2019) Physicsinformed neural networks: A deep learning framework for solving forward and inverse problems involving nonlinear partial differential equations. Journal of Computational Physics 378:686-707

Ramachandran P, Barret Z, Le QV (2018) Searching for activation functions. 6th International Conference on Learning Representations, ICLR 2018 - Workshop Track Proceedings

Sau R, Mahesh K (2010) Optimization of pulsed jets in crossflow. Journal of Fluid Mechanics 653:365-390

Schaeffler NW, Hepnery TE, Jones GS, Kegerise MA (2002) Overview of active flow control actuator development at NASA Langley research center. 1st Flow Control Conference

Soria J, Atkinson C (2008) Towards 3C-3D digital holographic fluid velocity vector field measurement - Tomographic digital holographic PIV (Tomo-HPIV). Measurement Science and Technology 19(7)

Sun K, Xiao B, Liu D, Wang J (2019) Deep highresolution representation learning for human pose estimation. In: CVPR California

Sun L, Gao H, Pan S, Wang JX (2020) Surrogate modeling for fluid flows based on physics-constrained deep learning without simulation data. Computer Methods in Applied Mechanics and Engineering 361

Szegedy C, Zaremba W, Sutskever I, Bruna J, Erhan D, Goodfellow I, Fergus R (2014) Intriguing properties of neural networks. 2nd International Conference on Learning Representations, ICLR 2014 - Conference Track Proceedings

Turing AM (1950) Computing Machinery and Intelligence. Parsing the Turing Test: Philosophical and Methodological Issues in the Quest for the Thinking Computer pp 23-65

Vernet R, Thomas L, David L (2009) Analysis and reconstruction of a pulsed jet in crossflow by multiplane snapshot POD. Experiments in Fluids 47

Williams DR, MacMynowski DG (2012) Brief History of Flow Control. Fundamentals and Applications of Modern Flow Control pp 1-20

Zaman KB, McKinzie DJ, Rumsey CL (1989) A Natural Low-Frequency Oscillation of the Flow over an Airfoil Near Stalling Conditions. Journal of Fluid Mechanics 202(403):403-442 


\section{Appendices}

\section{A1. Conditional approach details}

The four steps of the conditional approach are the following: The first step is the detection of the triggers used to synchronize the data with a common base time. The rising edges of the actuator square control signal are used here. This allows to check the forcing frequency, to determine the first trigger which will become the new time origin and the number of periods available for the processing. The second step consists to perform a classical phase-average $U_{\phi}$ over these periods. This process is illustrated in Fig.Ш, where $t^{*}$ is the dimensionless time $t / T$ (with $T$ the actuation period) and $U(t)$ is the velocity measured by the hot-wire for the instant $t$. The third step is the definition of the new common time origin stored in a $i \times j$ matrix, with $i$ the number of variables (coordinates, velocity, RMS...) and $j$ the number of time steps in a period defined by

$j=\frac{f_{\text {acquisition }}(\mathrm{Hz})}{f_{\text {actuator }}(\mathrm{Hz})}$

These steps are carried out on all of the $k$ measurement points. Each matrix is stored in the $i \times j \times k$ tensor, then the instantaneous flow fields can be obtained for each time step by extracting a $(i k)$ matrix. It is then possible to animate these snapshots in dimensionless time (along $j$ ) in order to observe the phase-averaged flow field dynamics using time-resolved flow field animations. This process is schematized on Fig. $\mathbb{\text { . }}$.

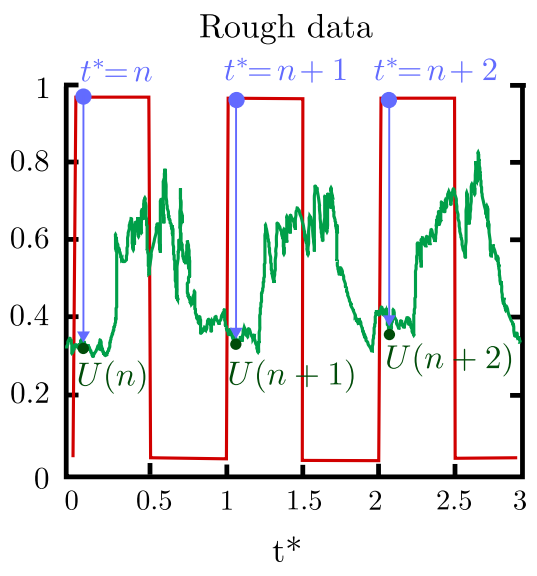

Phase-averaged data

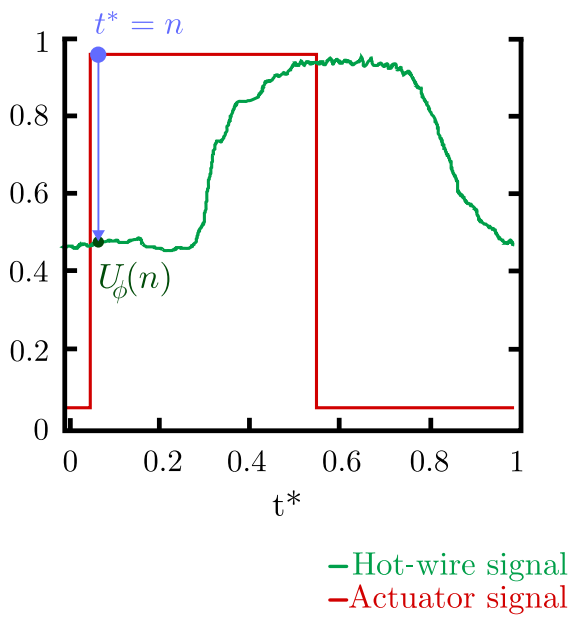

Fig. 7: Data set processing 
1

2 3 4 5 6 7 8 9 10

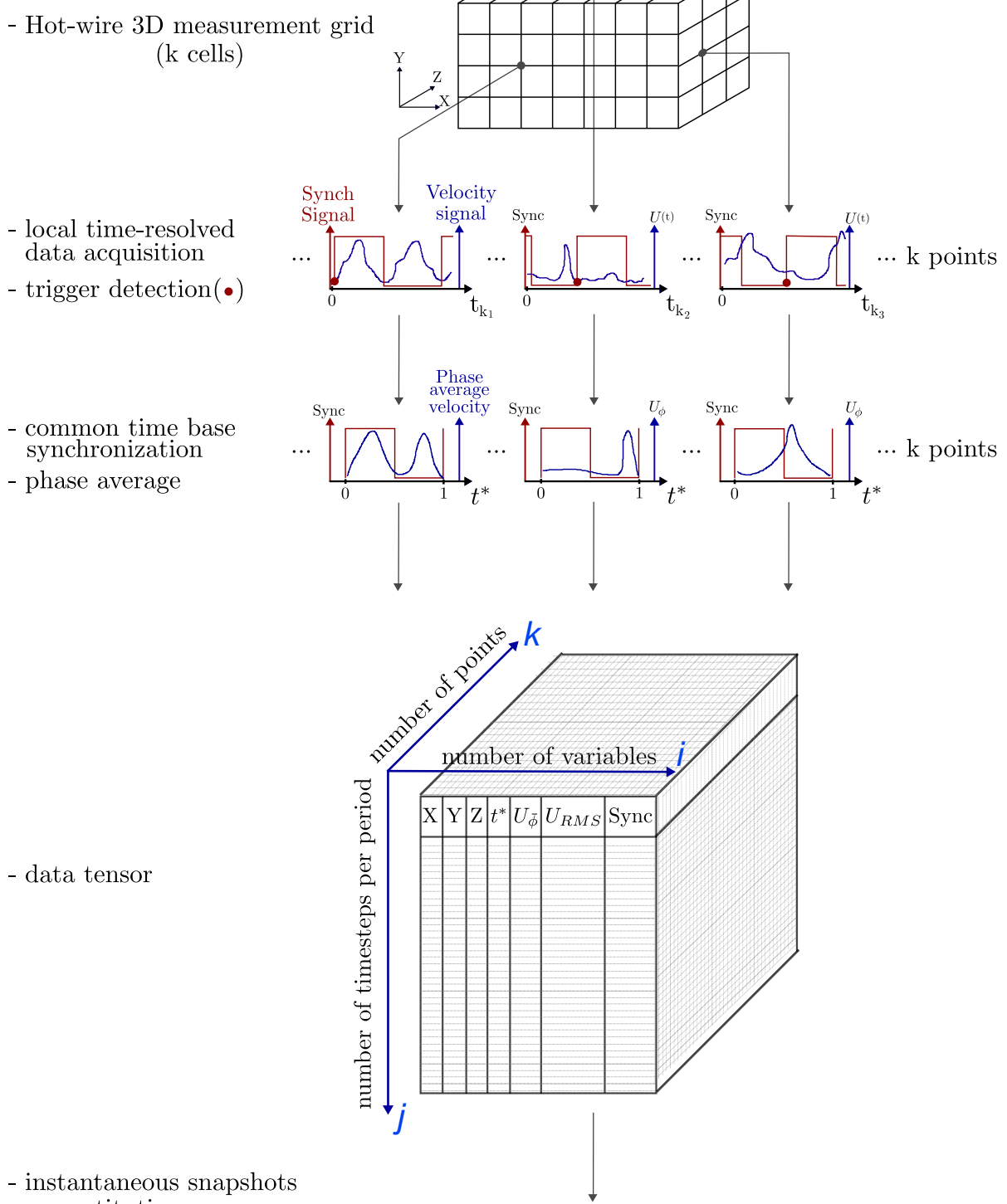

reconstitution
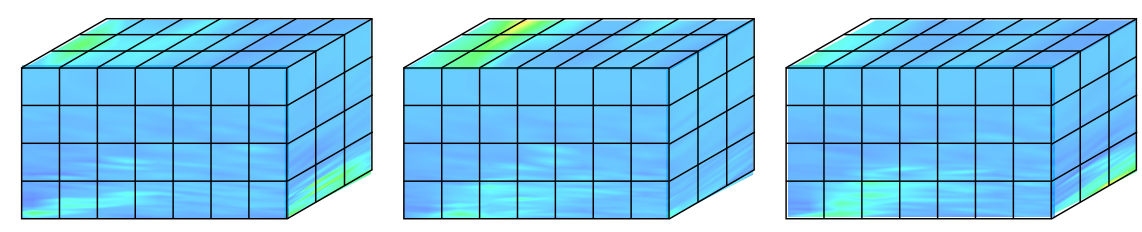

Fig. 8: 3D-adaped conditional approach Ott et al. (2019b) 
A2. Neural network: general introduction and terminology

In its simplest formulation, a neural network consists in interconnected units (called neurons) transforming the input information through a feed-forward process. One neuron and its associated parameters is shown in Fig.. . This neuron receives a weighted information $\Sigma=$

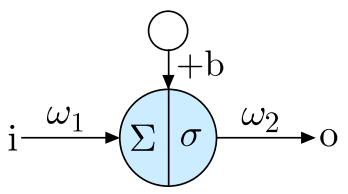

Fig. 9: Artificial neuron and its associated connections

$\omega_{1} \times i$ and activates according to the $\sigma$ function. The activation is classically a sigmoid reading:

$\sigma(\Sigma)=\frac{1}{1+\exp (-\Sigma)}$

Given a bias $b$ for the neuron and a weight $\omega_{2}$ for the output, the considered neuron transforms the input $i$ into $o=\omega_{2} \times \sigma\left(\omega_{1} \times i+b\right)$. In a dense network, each connection has a specific weight and each neuron has a specific bias. The Fig. tecture: one input layer with one neuron, one hidden layer with two neurons and one output layer with two neurons. The input value is $x$. In the hidden layer, the upper neuron outputs $\sigma\left(w_{1} \times x+b_{1}\right)$ while the lower neuron outputs $\sigma\left(w_{2} \times x+b_{2}\right)$. The neuron in the out-

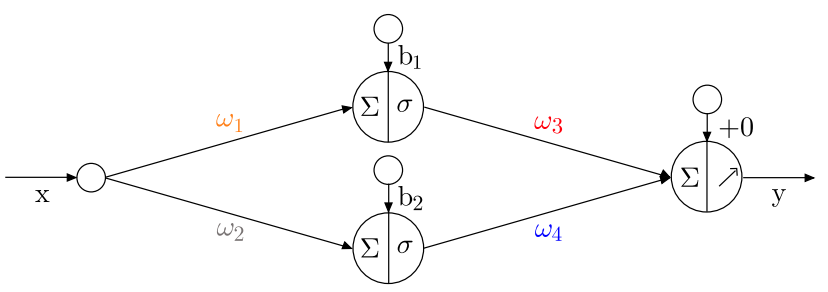

Fig. 10: Interactions between four neurons

put layer receives a weighted sum of these two outputs and linearly activates according to:

$y=w_{3} \times \sigma\left(w_{1} \times x+b_{1}\right)+w_{4} \times \sigma\left(w_{2} \times x+b_{2}\right)$

The final output then includes a sigmoid at $x=-b_{1} / w_{1}$ with an intensity of $w_{3}$ and another sigmoid at $x=$ $-b_{2} / w_{2}$ with an intensity of $w_{4}$. Just like a linear regression $y=a x+b$ would learn the best coefficients $a$ and $b$ from training data points $\left\{x_{i}, y_{i}\right\}$, a neural network $y=\mathrm{NN}(x)$ learns the optimal weights $w$ and biases $b$ to recover training examples from sigmoids.

These parameters are learned by minimizing a cost function which is often the mean square error between training data and their estimations. Considering $m$ training examples, the cost function then reads:

$E_{2}=\sum_{t=1}^{m}\left(y_{t}-\mathrm{NN}_{t}\right)^{2}$

Where $\mathrm{NN}_{t}=\mathrm{NN}\left(x_{t}\right)$. The neural network operator $N N$ is built upon parameters and hyperparameters. Parameters are optimized via a gradient descent on the cost function and hyperparameters (number of layers and number of neurons) are often based on the user experience. 